\title{
Direct numerical simulations of helical dynamo action: MHD and beyond
}

\author{
D. O. Gómez ${ }^{1, *}$ and P. D. Mininni ${ }^{2}$ \\ ${ }^{1}$ Departamento de Física, Facultad de Ciencias Exactas y Naturales, Universidad de Buenos Aires, Ciudad Universitaria, 1428 \\ Buenos Aires, Argentina \\ ${ }^{2}$ Advanced Study Program, National Center for Atmospheric Research, P.O. Box 3000, Boulder, Colorado 80307, USA \\ *also at: Instituto de Astronomía y Física del Espacio, CONICET, Ciudad Universitaria, 1428 Buenos Aires, Argentina
}

Received: 30 September 2004 - Revised: 10 November 2004 - Accepted: 12 November2004 - Published: 3 December 2004

Part of Special Issue "Advances in space environment turbulence"

\begin{abstract}
Magnetohydrodynamic dynamo action is often invoked to explain the existence of magnetic fields in several astronomical objects. In this work, we present direct numerical simulations of MHD helical dynamos, to study the exponential growth and saturation of magnetic fields. Simulations are made within the framework of incompressible flows and using periodic boundary conditions. The statistical properties of the flow are studied, and it is found that its helicity displays strong spatial fluctuations. Regions with large kinetic helicity are also strongly concentrated in space, forming elongated structures. In dynamo simulations using these flows, we found that the growth rate and the saturation level of magnetic energy and magnetic helicity reach an asymptotic value as the Reynolds number is increased. Finally, extensions of the MHD theory to include kinetic effects relevant in astrophysical environments are discussed.
\end{abstract}

\section{Introduction}

In magnetohydrodynamic (MHD) dynamos, an initially small magnetic field is amplified and sustained by currents induced solely by the motion of a conducting fluid (Moffatt, 1978). Dynamo action is often invoked to explain the existence of magnetic fields in several astronomical objects, including the Sun (Larmor, 1919; Parker, 1955; Leighton, 1969; Dikpati and Charbonneau, 1999), late-type stars (Brandenburg et al., 1998; Tobias, 1998), accretion disks (Blandford and Payne, 1982; Matsumoto et al., 1996; Casse and Keppens, 2004), and the Earth (Bullard, 1949; Braginsky, 1964; Glatzmaier et al., 1999; Buffett, 2000). Together with rotation, turbulence is ubiquitous in all these objects, and the generation of a magnetic field by a turbulent flow has become a crucial aspect of dynamo theory. Evidence of the

Correspondence to: D. O. Gómez

(dgomez@df.uba.ar) existence of turbulent flows in astrophysics can be found in a large number of objects, such as the interstellar medium (Armstrong et al., 1995; Minter and Spangler, 1996) or the solar convective region (Espagnet et al., 1993; Krishan et al., 2002).

In the last decades dynamo theory made significant advances, pushed forward by the positive feedback from direct numerical simulations (DNS), and the growing power of computers. The linear (or kinematic) regime of dynamo action was first studied theoretically within the framework of mean field magnetohydrodynamics (Steenbeck et al., 1966; Krause and Rädler, 1980). Later, its nonlinear regime was studied using MHD closures, such as the EDQNM (Pouquet et al., 1976). These studies led to the conclusion that a helical flow can under general conditions generate a large scale magnetic field through the $\alpha$-effect or the inverse cascade of magnetic helicity. Since then, direct simulations of MHD turbulence have given an important insight into the problem.

The first numerical simulation of dynamo action was made by Meneguzzi et al. (1981). Although computer resources were insufficient at the time to span magnetic diffusion timescales, the results made clear that a helical flow can amplify an initially small magnetic field exponentially fast, as well as significantly increase its spatial correlation. Later, several turbulent simulations were carried using mechanic helical forces (e.g. ABC type force), most of them with idealized conditions (Galanti et al., 1991; Brandenburg, 2001; Archontis et al., 2003; Mininni et al., 2003).

These simulations are often made in the incompressible MHD limit (see however Meneguzzi and Pouquet (1989); Cattaneo (1999); Balsara and Pouquet (1999) for studies of the effect of compressibility or stratification), and using periodic boundary conditions. Extensions of these models to include idealized boundaries can be found e.g. in Brandenburg and Dobler (2001). These conditions are far from astrophysical or geophysical applications, but allow drastic 
simplifications in the equations and numerical methods. In turn, these simplifications allow to resolve a larger separation of scales, which is crucial to study turbulent dynamo action. Actually, the strongest limitation for DNS is related with the extreme computational resources needed to ensure this scale separation. A systematic study of different flows, long simulations, or simulations in the range of parameters found in astrophysics are impossible in the foreseeable future. As a result, it seems evident that theory and simulations must evolve together to reach a deeper understanding of the problem.

On the other hand, in DNS the conditions that the flow must satisfy to amplify and sustain a magnetic field can be studied directly. In this work we follow this path and review recent results in the study of helical dynamo action. In Sect. 2 we introduce the MHD equations, and in Sect. 3 we present results for the time evolution of the MHD helical dynamo. In the last years, new effects relevant in several astrophysical scenarios have been introduced into the theory and simulations. Section 4 reviews a few of these extensions, and discuss recent results found within the framework of HallMHD. Finally, in Sect. 5 we present the conclusions of this work.

\section{The equations}

Incompressible MHD is described by the induction and the Navier-Stokes equations,

$$
\begin{aligned}
\frac{\partial \boldsymbol{B}}{\partial t}= & \nabla \mathbf{x}(\boldsymbol{U} \times \boldsymbol{B})+\eta \nabla^{2} \boldsymbol{B} \\
\frac{\partial \boldsymbol{U}}{\partial t}= & -(\boldsymbol{U} \cdot \nabla) \boldsymbol{U}+(\boldsymbol{B} \cdot \nabla) \boldsymbol{B}- \\
& -\nabla\left(P+\frac{B^{2}}{2}\right)+\boldsymbol{F}+v \nabla^{2} \boldsymbol{U},
\end{aligned}
$$

with the additional constraints $\nabla \cdot \boldsymbol{U}=\nabla \cdot \boldsymbol{B}=0$. The external force $\boldsymbol{F}$ is assumed to be solenoidal, and the velocity $\boldsymbol{U}$ and magnetic field $\boldsymbol{B}$ are expressed in units of a characteristic speed $U_{0}$. The pressure $P$ was divided by the constant fluid density. Here, $\eta$ and $v$ are respectively the magnetic diffusivity and the kinematic viscosity (assumed constant and uniform).

The MHD system has three ideal quadratic invariants (i.e. for $\eta=\nu=0$ )

$$
\begin{aligned}
E & =\frac{1}{2 V} \int_{V}\left(U^{2}+B^{2}\right) d V=\frac{1}{2}\left\langle U^{2}+B^{2}\right\rangle, \\
H_{m} & =\frac{1}{2 V} \int_{V} \boldsymbol{A} \cdot \boldsymbol{B} d V=\frac{1}{2}\langle\boldsymbol{A} \cdot \boldsymbol{B}\rangle, \\
H_{c} & =\frac{1}{2 V} \int_{V} \boldsymbol{B} \cdot \boldsymbol{U} d V=\frac{1}{2}\langle\boldsymbol{B} \cdot \boldsymbol{U}\rangle .
\end{aligned}
$$

Here $E$ is the mean energy density, $H_{m}$ is the mean magnetic helicity, and $H_{c}$ is the mean cross helicity. It is standard practice in numerical simulations to compute the volume average of the ideal invariants over the total volume $V$ (indicated by $\langle\ldots>$ ), rather than their total values. The vector potential $\boldsymbol{A}$ is defined by $\boldsymbol{B}=\nabla \times \boldsymbol{A}$.

The simulations discussed in the following sections were made using a parallel pseudospectral code (Mininni et al., 2003, 2004; Gómez et al., 2004). Equations (1) and (2) are integrated in a cubic box with periodic boundary conditions. The code implements Runge-Kutta of several orders to evolve the equations in time, but most of the simulations were performed using Runge-Kutta of order two. The total pressure $P_{T}=P+B^{2} / 2$ is computed solving a Laplace equation in Fourier space at each time step, to ensure the incompressibility condition $\nabla \cdot \boldsymbol{U}=0$ (Canuto et al., 1988). To satisfy the divergence-free condition for the magnetic field, the induction equation (1) is replaced by an equation for the vector potential

$\frac{\partial \boldsymbol{A}}{\partial t}=\boldsymbol{U} \times \boldsymbol{B}-\nabla \phi+\eta \nabla^{2} \boldsymbol{A}$,

where $\phi$ (the self-consistent electrostatic potential) is computed at each time step to satisfy the gauge $\nabla \cdot \boldsymbol{A}=0$, by solving another Laplace equation with the same method used to obtain the pressure. The code uses the 2/3-rule to control aliasing error (Canuto et al., 1988), and therefore the largest wavenumber $k_{\max }$ that can be resolved in a spatial grid of $N^{3}$ points is given by $k_{\max }=N / 3$. In this paper, we show simulations up to resolutions of $256^{3}$ grid points.

\section{MHD helical dynamos}

It is clear from the equations presented in the previous section, that effects such as stratification, magnetic buoyancy, or rotation are neglected. As a result, no natural mechanism is present to break the mirror symmetry in the flow. Therefore, a net kinetic helicity must be injected into the fluid by the body force $\boldsymbol{F}$. Usually, eigenfunctions of the curl operator are used to force the fluid, ensuring both injection of energy and kinetic helicity at large scales. To help scale separation, the energy injection band is often restricted to a few wave numbers around the wavenumber $k_{\text {force }}$. In our simulations we use an $\mathrm{ABC}$ force $\boldsymbol{F}$ with $A=B=C$ (Childress, 1970), acting at $k_{\text {force }}$ (see Mininni et al. (2003) for details).

The amount of helicity in the flow is measured by the kinetic helicity (Moffatt, 1978)

$H_{k}=\frac{1}{2 V} \int_{V} \boldsymbol{U} \cdot \boldsymbol{\omega} d V=\frac{1}{2}\langle\boldsymbol{U} \cdot \boldsymbol{\omega}\rangle$,

where $\omega=\nabla \times \boldsymbol{U}$ is the vorticity. It is also useful to normalize this quantity between 1 and -1 introducing the relative helicity $2 H_{k} /\left(\left\langle U^{2}\right\rangle\left\langle\omega^{2}\right\rangle\right)^{1 / 2}$.

The main question is whether a turbulent flow can amplify and sustain a magnetic field. As a result, no source of magnetic field is used except for a small amplitude random seed at scales smaller than the energy injection band.

\subsection{Helical flows}

Before introducing the magnetic seed, a fully developed turbulent flow is needed. To this end, a forced hydrodynamic 

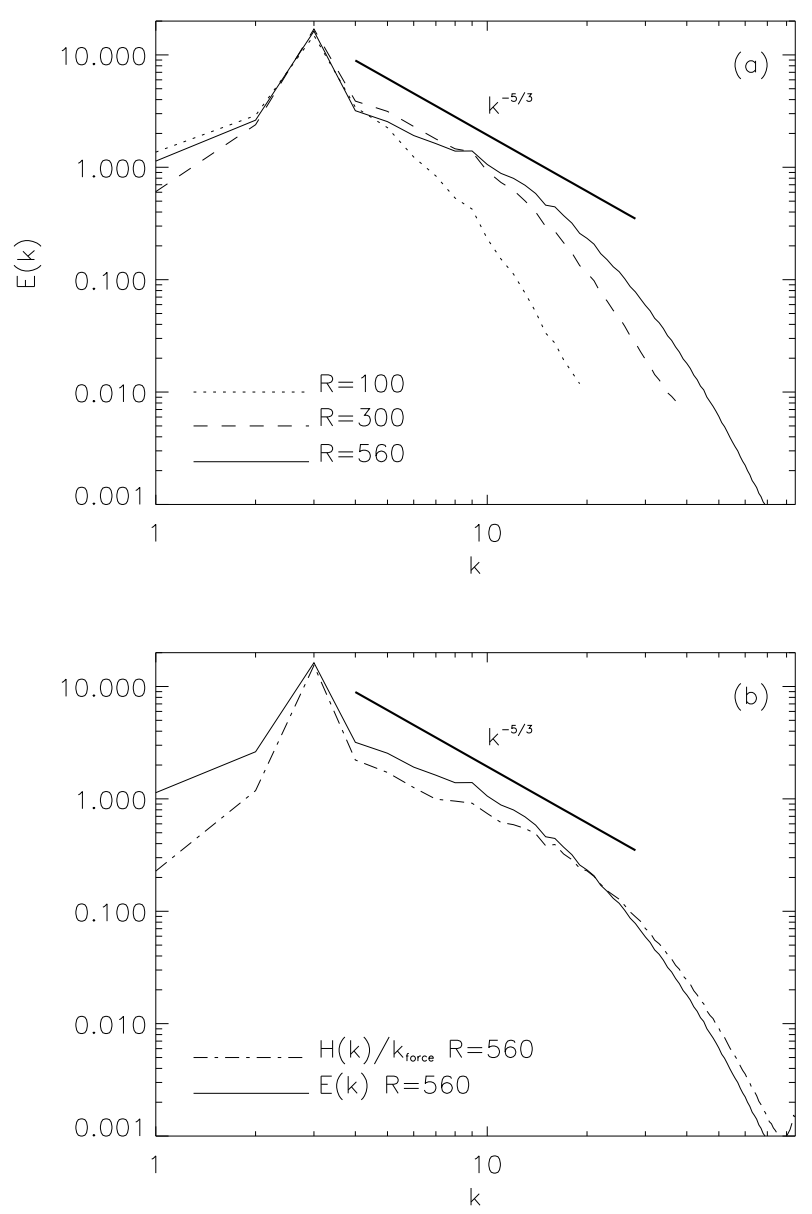

Fig. 1. (a) Kinetic energy spectrum at different Reynolds numbers. (bfb) Spectrum of kinetic energy, and kinetic helicity divided by $k_{\text {force }}$. The Kolmogorov's law is showed as a reference.

simulation is carried out until it reaches a statistically steady state. The resulting flow has, say, positive helicity (in all the simulations discussed here this will be the case). The r.m.s value of the velocity $U_{0}=\left\langle U^{2}\right\rangle^{1 / 2}$ is used to define the Reynolds number $R=U_{0} L_{0} / \nu$. The value of $R$ in all our simulations is well above the threshold $\left(R^{c} \approx 50\right)$ in which the ABC flow destabilizes (Podvigina and Pouquet, 1994).

We will first study the hydrodynamic properties of the flow, since when the small magnetic field is introduced the Lorentz force $\boldsymbol{J} \times \boldsymbol{B}$ in Eq. (2) can be neglected. Equation (1) is linear in the magnetic field, and the electromotive force $\boldsymbol{U} \times \boldsymbol{B}$ is the only term able to amplify $\boldsymbol{B}$. Therefore, the geometrical properties of the flow are directly responsible for the observed amplification.

Figure 1a shows the kinetic energy spectrum $E_{k}(k)$ in a hydrodynamic simulation for different values of $R$ and $k_{\text {force }}=3$. As the Reynolds number is increased, a scale separation between the energy injection wavenumber and the dissipation wavenumber appears. Then, three regions can be identified in Fourier space. The energy injection band, the inertial range, and the dissipation range. The energy is injected
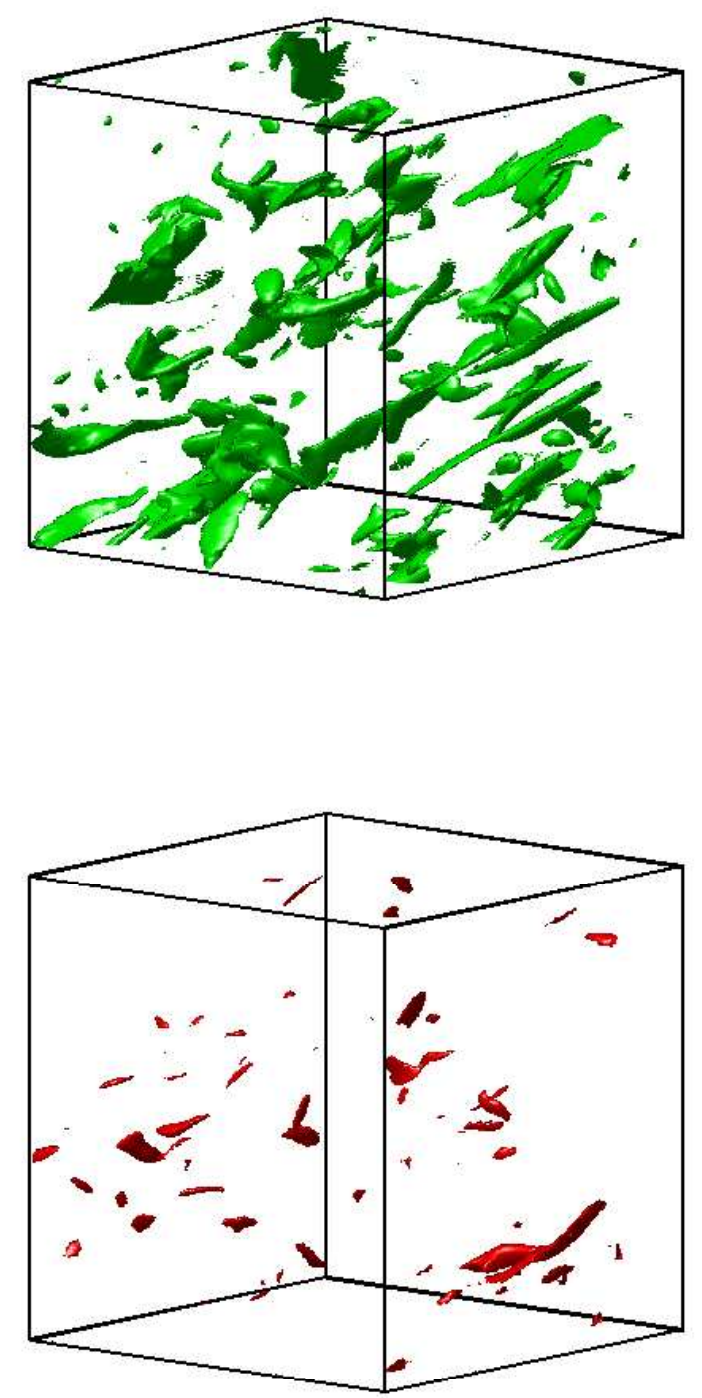

Fig. 2. Isosurfaces of kinetic helicity (at $20 \%$ of the maximum value) in a helical hydrodynamic simulation with $256^{3}$ grid points $\left(R=560, k_{\text {force }}=3\right.$ ). Green (above) indicates positive helicity, while red (below) corresponds to negative helicity. Note the presence of elongated structures. Both snapshots correspond to the same time. The region displayed is $1 / 8$ of the box.

at $k_{\text {force }}$, cascades down to smaller scales through the inertial range (which follows a Kolmogorov's $k^{-5 / 3}$ law), and finally dissipates at small scales. The dissipation wavenumber scales as $k_{\nu}=\left(\left|\omega^{2}\right\rangle / \nu^{2}\right)^{1 / 4}$. In all the simulations, this wavenumber is smaller than $k_{\max }$ and all scales in the flow are well resolved.

The kinetic helicity injected at $k_{\text {force }}$ also cascades down to smaller scales, and its spectrum is proportional to $k_{\text {force }} E(k)$. The direct cascade of kinetic helicity was expected from theoretical arguments (Brissaud et al., 1973; Kraichnan, 1973; Moffatt, 1978), and verified only recently in DNS (Chen et al., 2003; Chenet et al., 2003; Gómez and Mininni, 2004). Note that the kinetic helicity spectrum is not a positive defined quantity (Fig. 1b). However, as positive 


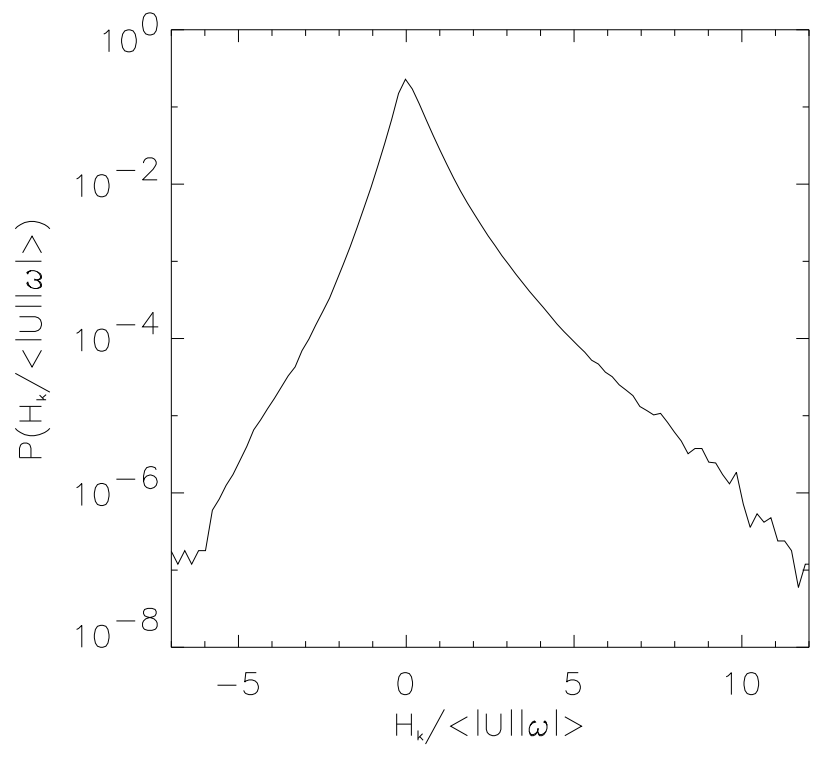

Fig. 3. PDF of the spatial density of kinetic helicity normalized by the r.m.s. values of velocity and vorticity, in the same simulation ( $256^{3}$ grid points, $R=560$ ). Note that although Schwartz inequality ensures that the total relative helicity is bounded between -1 and 1 , point to point this relation does not hold.

helicity is injected at $k_{\text {force }}$, all the wavenumbers up to $k_{v}$ are dominated by positive helicity. This does not imply that the flow in real space has positive helicity at every point (see Fig. 2). The nonlinear terms in the Navier-Stokes equation generates patches with different relative helicities. Indeed, the instantaneous flow shows regions with strong positive helicity isotropically distributed in space, and also regions with negative helicity. The filling factor of the positive regions is larger, and as a result positive helicity dominates at all scales. Helical regions display an elongated structure in real space.

The fluctuations of kinetic helicity can also be observed in its probability density function (PDF), shown in Fig. 3. The PDF is centered around zero, but it is asymmetric and has a stronger tail in the positive direction. As a result, regions with strong positive helicity have a larger probability than regions with strong negative helicity. The mean value of relative kinetic helicity in this simulation is 0.4.

Although the spectra of energy and kinetic helicity are proportional, the spectrum of relative helicity (not shown, see for instance Borue and Orszag (1997)) follows a $k^{-1}$ law, and large scales are relatively more helical than small scales.

\subsection{Kinematic regime}

Once the hydrodynamic steady state is reached, a random and non-helical magnetic field is introduced. The initial magnetic seed is usually generated by a $\delta$-correlated vector potential, centered at $k_{\text {seed }}$. This wavenumber is chosen to be smaller than the dissipation wavenumber, but larger than $k_{\text {force }}$. Its amplitude must be much smaller than the kinetic energy, at all scales. Then the simulation is continued with the same

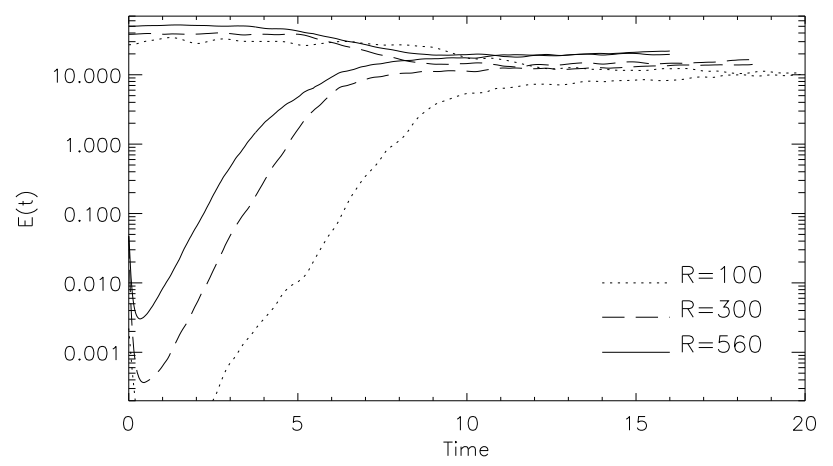

Fig. 4. Kinetic (above) and magnetic energy (below) as a function of time, for simulations with different Reynolds numbers $\left(k_{\text {force }}=3\right)$.

external helical force in the Navier-Stokes equation, to study the growth of magnetic energy due to dynamo action.

In a flow capable of dynamo action, amplification only takes place when the magnetic Reynolds number $R_{m}={ }_{0} L_{0} / \eta$ is larger than a critical value. For helical flows this threshold is small ( $R^{c} \approx 1$, see Galanti et al. (1992); Brandenburg (2001); Brandenburg and Subramanian (2004)), and all the simulations we consider are well above the critical case. The simulations we show are also for magnetic Prandtl number $P_{m}=v / \eta=1$, and therefore $R_{m}=R$.

Under these conditions, after a short time while magnetic energy is redistributed in Fourier space, the magnetic field grows exponentially as a function of time. Figure 4 shows the magnetic and kinetic energy as a function of time for simulations with different Reynolds numbers and $k_{\text {force }}=3$. During this kinematic stage, the r.m.s. value for the velocity is $U_{0}=\left\langle U^{2}\right\rangle^{1 / 2} \approx 6$. The turnover time for the energy containing eddies $\left(k_{\text {force }}=3\right)$ is then approximately equal to $\Delta t=0.3$. Therefore, as shown in Fig. 4, the kinematic regime lasts for about ten to twenty turnover times. Note also that the growth rate during the kinematic regime approaches an asymptotic value as $R$ is increased (Archontis et al., 2003; Mininni et al., 2004).

In this first stage, the magnetic energy is still weak and the velocity field is not strongly affected by the Lorentz force. As a result, during the exponential growth of magnetic energy the kinetic energy remains approximately constant. The evolution in the kinematic regime can be explained using the mean field induction equation (Steenbeck et al., 1966; Krause and Rädler, 1980)

$$
\frac{\partial \overline{\boldsymbol{B}}}{\partial t}=\nabla \times(\overline{\boldsymbol{U}} \times \overline{\boldsymbol{B}}+\alpha \overline{\boldsymbol{B}})+\eta_{e f f} \nabla^{2} \overline{\boldsymbol{B}} .
$$

Here the overline denotes mean field quantities (defined using an average satisfying Taylor's hypothesis), and $\eta_{\text {eff }}$ is the effective turbulent diffusivity. The $\alpha$-effect (Pouquet et al., 1976)

$\alpha=\frac{\tau}{3}(-\overline{\boldsymbol{u} \cdot \boldsymbol{\omega}}+\overline{\boldsymbol{b} \cdot \boldsymbol{j}})$, 


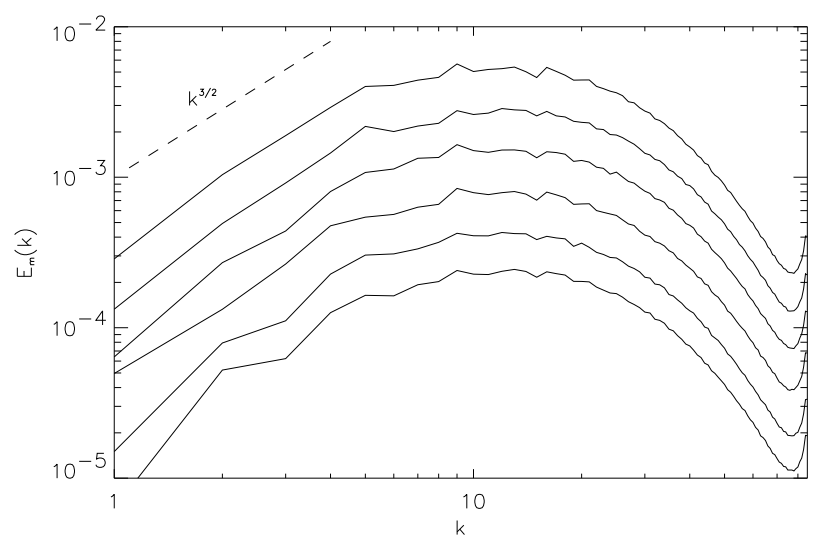

Fig. 5. Spectrum of magnetic energy during the kinematic regime, in a simulation with $k_{\text {force }}=3$ and $R=560\left(256^{3}\right.$ grid points $)$. Starting at $t=1$ (below) up to $t=2.5$ (above), the time interval between different spectra is $\Delta t=0.3$. All the modes grow with the same growth rate. The time $t$ corresponds to the one showed in Figs. 4 and 7. The Kazantsev slope $k^{3 / 2}$ is displayed for reference.

represents the back-reaction of the turbulent motions in the mean field. Here $\boldsymbol{u}$ and $\boldsymbol{b}$ are respectively the small-scale velocity and magnetic fields, $\boldsymbol{\omega}=\nabla \times \boldsymbol{u}$ and $\boldsymbol{j}=\nabla \times \boldsymbol{b}$ are the small-scale vorticity and current, and $\tau$ is a typical correlation time for these turbulent motions. Attempts to measure this quantity in direct simulations using different methods can be found in Cattaneo and Hughes (1996), and Brandenburg (2001). In the absence of a mean flow $\overline{\boldsymbol{U}}$, Eq. (9) predicts an exponential growth above a certain threshold if $\alpha \neq 0$ (as it is the case in helical turbulence).

In the kinematic regime, all the magnetic modes grow with the same rate (Fig. 5), and the spectrum of magnetic energy is peaked at small scales. As $R$ is increased, the peak in the spectrum moves to smaller scales (Fig. 6), but the ratio between the amplitudes of the large scale modes $(k=1,2)$ is virtually unchanged. The Kazantsev spectrum (Kazantsev, 1968), which corresponds to a random velocity field $\delta$ correlated in time, is displayed in Fig. 5 for reference. Although the hypothesis behind this spectrum are not satisfied, a qualitative agreement can be observed, as has also been reported by Haugen et al. (2004).

Magnetic helicity (Fig. 7) also grows exponentially during the kinematic regime, and with a sign which is opposite to the one of the kinetic helicity of the flow. This growth of magnetic helicity can also be explained using the $\alpha$-effect. From Eqs. (1, 4 and 6), the net magnetic helicity is conserved except for magnetic diffusion events at small scales

$\frac{d H_{m}}{d t}=-2 \eta \int \boldsymbol{J} \cdot \boldsymbol{B} d^{3} x$.

But at large scales the $\alpha$ effect can inject helicity on the mean field (Seehafer, 1996). From Eq. (8) we obtain for the time evolution of the mean field magnetic helicity

$\frac{d \overline{H_{m}}}{d t}=2 \int\left(\alpha \bar{B}^{2}-\eta_{e f f} \overline{\boldsymbol{J}} \cdot \overline{\boldsymbol{B}}\right) d^{3} x$.

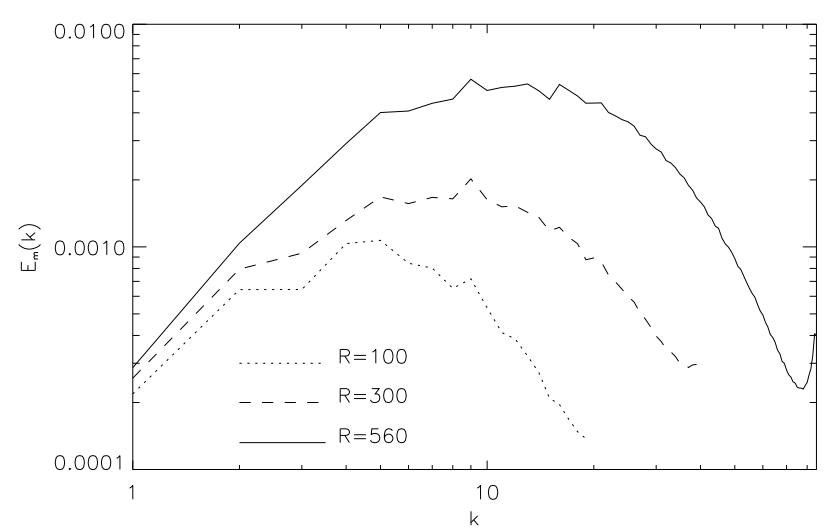

Fig. 6. Spectrum of magnetic energy during the kinematic regime $(t=2)$, in simulations with $k_{\text {force }}=3$ and different Reynolds numbers.

This equation represents a transfer of magnetic helicity from small scales to large scales. Since the net magnetic helicity is conserved, and the initial helicity is zero, magnetic helicity of different signs is segregated in different spatial scales, or different wavenumbers in Fourier space. The $\alpha$-effect is responsible for this process. It creates equal but opposite amounts of magnetic helicity in the small and large scales. The spectra of magnetic helicity confirm this (Fig. 8). But magnetic diffusion destroys the small scale magnetic helicity in reconnection events, leaving a net helicity of opposite sign at large scales (Brandenburg, 2001).

Note that from Eq. (11) the mean magnetic helicity has the same sign as the $\alpha$ coefficient, and therefore (from Eq. 9) the sign opposite to that of kinetic helicity. The signs shown in Fig. 7 are in good agreement with this relation.

This effect is expected to decrease as the magnetic Reynolds number $R_{m}$ is increased. Moreover, several authors claim that the total magnetic helicity generated by the $\alpha$-effect must approach zero in the high $R_{m}$ limit (Gilbert, 2002; Hughes et al., 1996; Brandenburg and Subramanian, 2004). However, as it is also observed in the growth of magnetic energy, the growth rate of magnetic helicity during the kinematic regime approaches an asymptotic value as $R$ is increased (Mininni et al., 2004).

\subsection{Saturation of the dynamo}

When the Lorentz force is strong enough, saturation takes place and the magnetic energy stops growing exponentially (see Fig. 4). Since the kinetic energy spectrum follows a power law $k^{-5 / 3}$, and during the kinematic regime all the magnetic modes grow with the same rate peaking at small scales (Figs. 5 and 6), saturation takes place first at the small scales. This was predicted by Pouquet et al. (1976) using the EDQNM closure. Indeed, Eq. (9) shows that the $\alpha$-effect is quenched by the term $\overline{\boldsymbol{j} \times \boldsymbol{b}}$, proportional to the current helicity at small scales. 


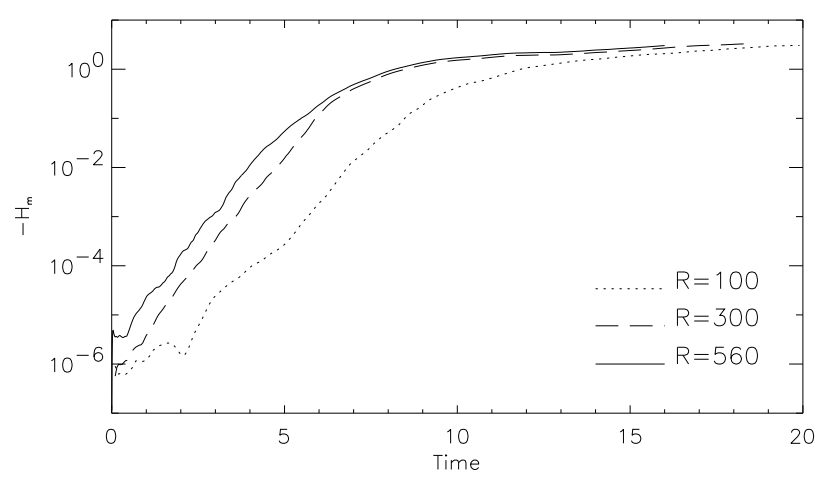

Fig. 7. Evolution of magnetic helicity (negative), in dynamo simulations with different Reynolds numbers $\left(k_{\text {force }}=3\right)$.

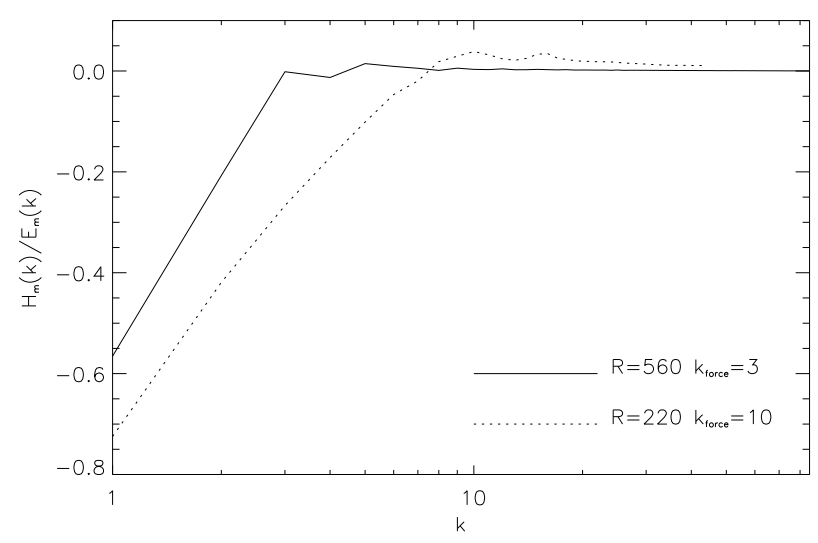

Fig. 8. Spectrum of magnetic helicity at $t=5$, normalized by the magnetic energy at each $k$. Note the peak and the change in sign close to $k_{\text {force }}$. Large scales are dominated by negative magnetic helicity, while the kinetic helicity of the flow is positive at all scales (see e.g. Fig. 1b).

During and after the saturation, the magnetic field is stronger than the velocity field at small scales (Fig. 9). Note that the kinetic energy spectrum at small scales is quenched by the Lorentz force, and therefore velocity fluctuations at small scales are suppressed by the growing magnetic field. The kinetic energy spectrum deviates from the Kolmogorov's law, and its slope is steeper than $-5 / 3$ (see Fig. 9b).

This effect can be seen more clearly as the Reynolds number and the scale separation between the energy injection band and the small scales are increased (Mininni et al., 2004). Figure 10 shows the compensated energy spectrum $E(k) k^{5 / 3} \epsilon^{-2 / 3}$ in a simulation with $k_{\text {force }}=3$ and $R=560$, where $\epsilon$ is the energy injection rate. If the energy spectrum $E(k)$ follows a Kolmogorov's law in the inertial range

$E(k)=C_{K} \epsilon^{2 / 3} k^{-5 / 3}$,

then the compensated spectrum should be flat in this range, and its amplitude corresponds to the Kolmogorov's constant $C_{K}$.
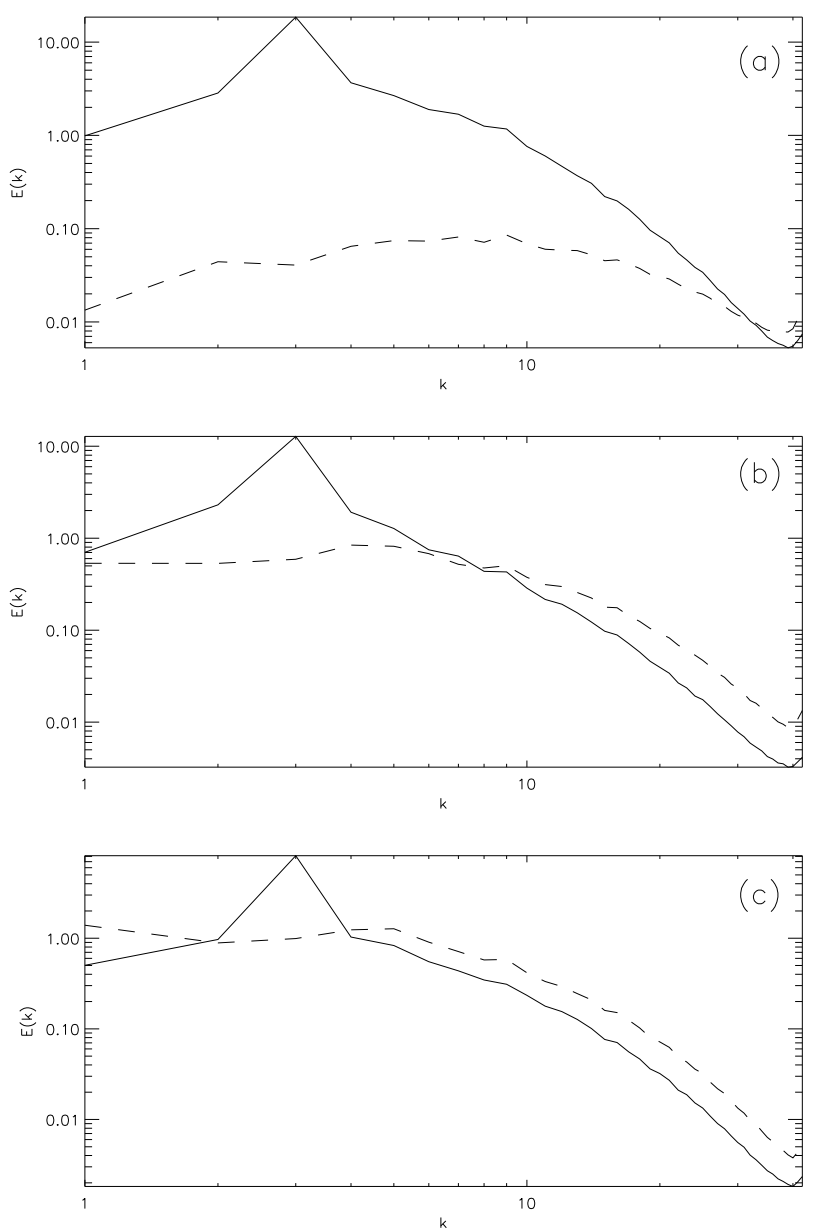

Fig. 9. Spectrum of kinetic (solid lines) and magnetic energy (dashed lines) during saturation at small scales, in a simulation with $k_{\text {force }}=3$ and $R=300$ : (a) $t=5$, (b) $t=7$, and (c) $t=9$.

As can be seen from Fig. 10, small scales are dominated by the magnetic energy, and the kinetic energy spectrum is steeper than $-5 / 3$ during the saturation. However, the total energy spectrum follows a Kolmogorov's law. This power law is satisfied by the total energy during the complete evolution of the dynamo (kinematic regime, saturation, and final state).

\subsection{Large scale magnetic field}

Note that the saturation of the magnetic energy spectrum is only partial (Fig. 9). The magnetic field at small scales stops to grow. But if there is enough scale separation between $k_{\text {force }}$ and the largest scale in the box $(k=1)$, the magnetic energy at large scales keeps growing slowly (not exponentially), as shown in Fig. 11. While no changes can be observed in the magnetic spectrum at wavenumbers larger than $k_{\text {force }}$, the amplitude of the magnetic field in the shell $k=1$ increases with time. The effect is more clear as $k_{\text {force }}$ is increased, and gives rise to a slow saturation (Brandenburg, 2001; Brandenburg and Dobler, 2002) of the total magnetic 


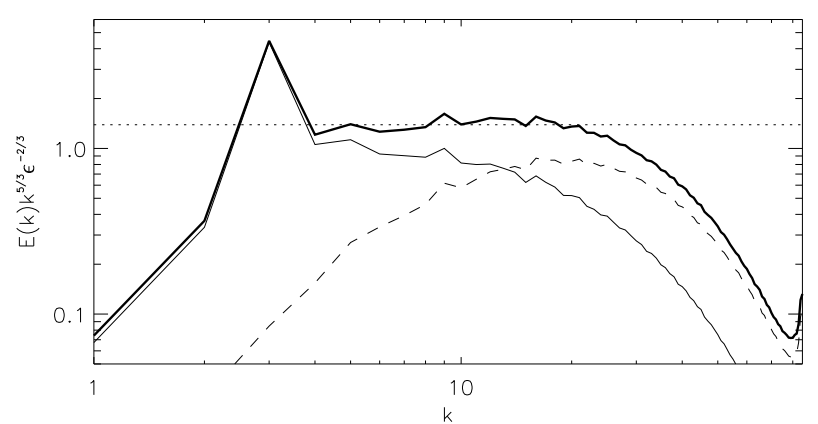

Fig. 10. Compensated spectrum of kinetic, magnetic and total energy after saturation $(t=6)$, in a simulation with $k_{\text {force }}=3$ and $R=560$.

energy (see Fig. 4). The increase of magnetic energy at large scales can also be observed in real space (Fig. 12).

The large scale magnetic field $(k=1)$ is an approximately force-free field. Neglecting the mean flow $\overline{\boldsymbol{U}}$, assuming a solution of the form

$\overline{\boldsymbol{B}}=\boldsymbol{B}_{0} e^{i \boldsymbol{k}_{0} \cdot \boldsymbol{x}+\sigma t}$,

and replacing in the mean field Eq. (8), we finally obtain

$i \alpha \boldsymbol{k}_{0} \times \boldsymbol{B}_{0}=\left(\sigma+\eta_{e f f} k^{2}\right) \boldsymbol{B}_{0}$

This equation is satisfied if $\boldsymbol{B}_{0}$ is an eigenvector of the curl operator, $\nabla \times \boldsymbol{B}_{0}= \pm k_{0} \boldsymbol{B}_{0}$. Note that in this case the Lorentz force $\boldsymbol{J} \times \boldsymbol{B}$ at large scales is zero, and the hypothesis of negligible mean flow is consistent with the solution. Equation (14) only holds during the kinematic regime. However Fig. 11b shows that in the saturated state the spectrum at large scales is dominated by magnetic energy as $k_{\text {force }}$ is increased, and Meneguzzi et al. (1981) and Brandenburg (2001) verified in DNS of helical dynamo action that the magnetic field at $k=1$ is a force-free field.

Using this result, and the asymptotic conservation of magnetic helicity from Eq. (10), Brandenburg (2001) showed that the saturation of the amplitude of the large scale magnetic field is achieved as

$\bar{B}(t)=B_{0}\left[1-e^{-2 \eta k_{0}^{2}\left(t-t_{s a t}\right)}\right]$.

The time evolution of the amplitude of the large scale magnetic field from DNS shows a good agreement with this relation (Brandenburg, 2001). Equation (15) indicates that the saturation of the large scale field takes place in a resistive time scale, and is dominated by the evolution of magnetic helicity.

Due to this build up of a coherent magnetic field with a correlation length of the size of the box, helical dynamos (considered as dynamos produced by flows mostly dominated by one sign of kinetic helicity, as discussed at the beginning of this section) are often called "large scale dynamos". This name is used in the literature in opposition to "small scale dynamos" where a non-helical flow is studied (Kazantsev,
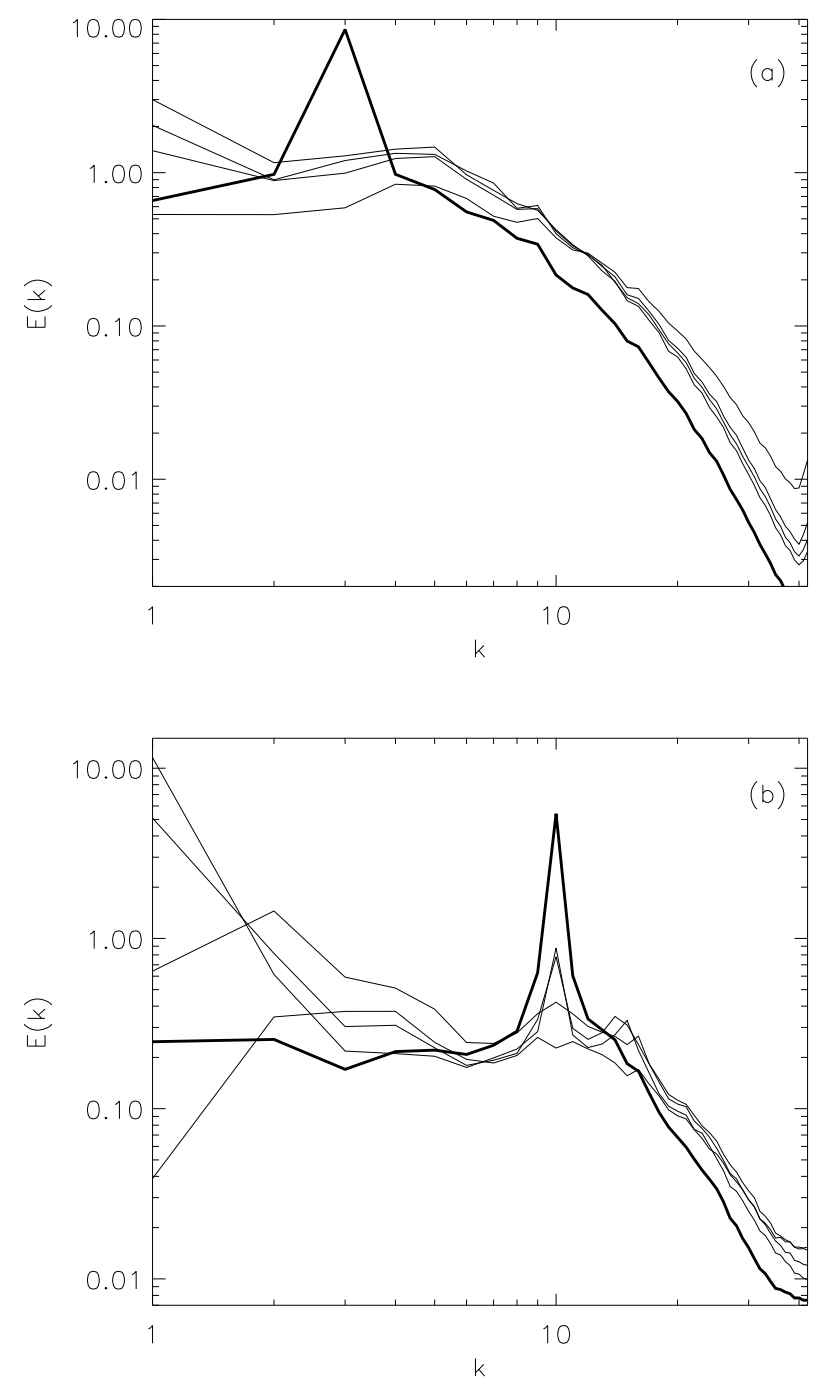

Fig. 11. Magnetic energy spectrum (thin lines) as a function of time (from $t=7$ up to $t=20$, time as in Fig. 4), and kinetic energy spectrum at late times (thick line), during the last stage of the saturation of the dynamo: (a) $k_{\text {force }}=3$ and $R=300$, (b) $k_{\text {force }}=10$ and $R=220$. Note the slow increase of magnetic energy at $k=1$.

1968; Zeldovichet al., 1983; Schekochihin et al., 2001, 2004; Haugen et al., 2003), and the magnetic field generated is correlated on scales much smaller than the energy containing scales of the turbulence. However, we want to point out that a large scale magnetic field can in some cases be generated without fully helical turbulence or a net $\alpha$-effect. Anisotropic flows can generate large scale magnetic fields (Nore et al., 1997). As another example, in mean field theory there are terms in the mean electromotive force that can build a mean field when the $\alpha$ coefficient is zero (Urpin, 2002). The $\boldsymbol{\omega} \times \boldsymbol{j}$ term in this expansion is also known to generate large scale magnetic fields (Geppert and Rheinhardt, 2002). 


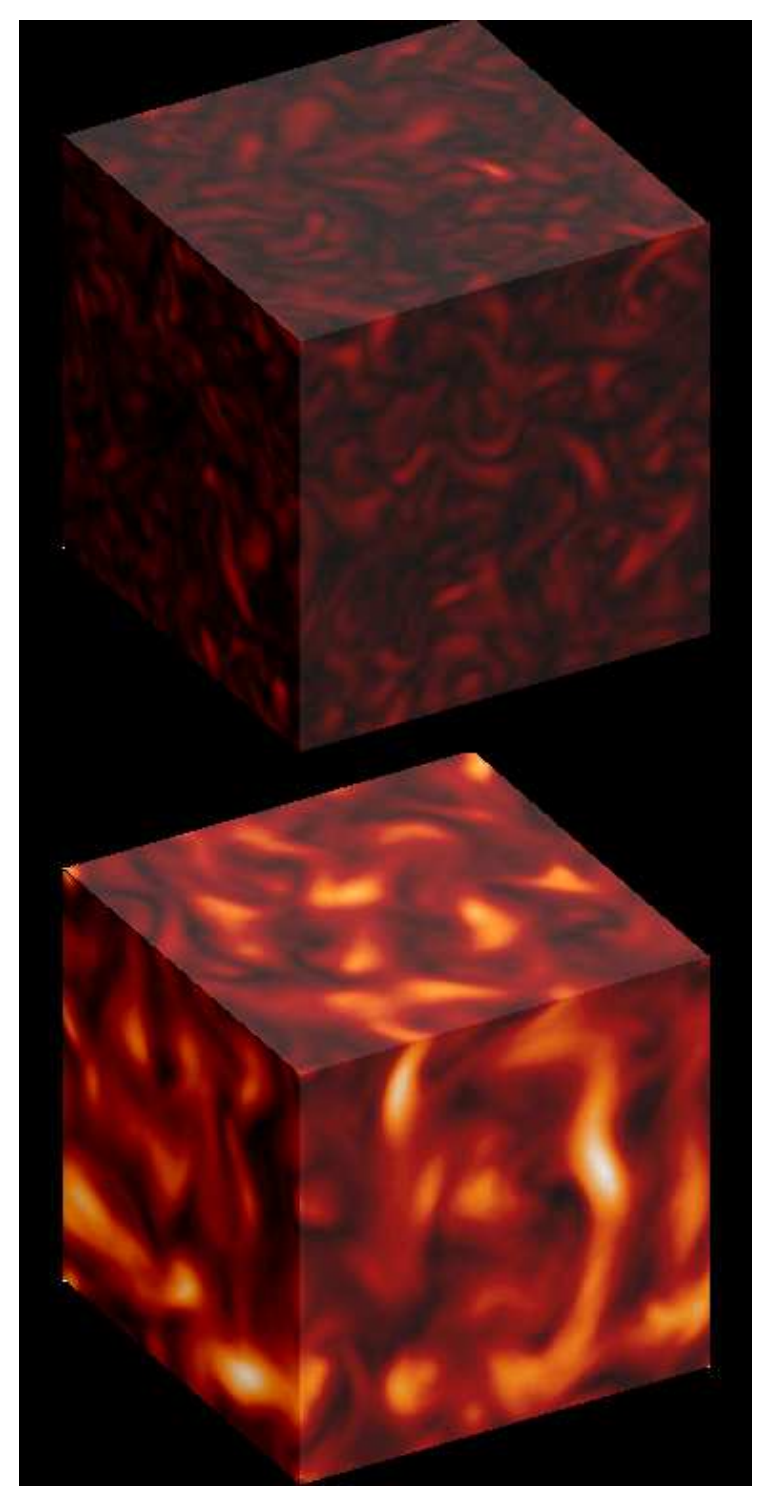

Fig. 12. Spatial density of magnetic energy in a MHD dynamo simulation, at early times (above) and during the saturation (below). The increase in the amplitude of the field and in the correlation length can be observed.

\section{Beyond the MHD approximation}

One-fluid MHD, as presented in Eqs. (1 and 2) and used in the previous section, is the standard framework used for describing dynamo action in astronomical objects. It is a good approximation for the solar dynamo and the geodynamo (Priest and Forbes, 2000). Also, one-fluid MHD often turns out to be a reasonable description of the large scale bulk dynamics of the fluid, as long as the fluid does not support a significant electric field in its own frame of reference. In idealized DNS of dynamo action, MHD is often used to obtain a larger scale separation, since the introduction of two-fluid effects would increase the computer resources needed to carry out the simulations.
The increase of computing power in the last years allowed the study of some extensions to the MHD dynamo theory, relevant in some astrophysical scenarios characterized by low temperatures, partial ionization, or collisionless plasmas. MHD cannot be expected to properly describe these plasmas because it fails to distinguish the relative motions between different species. A first step toward creating a more appropriate theory for these objects might be to include the dominant two-fluid effects considering a generalized Ohm's law. The most relevant effects for astrophysical applications are ambipolar diffusion and the Hall effect (Zweibel, 1988; Wardle, 1999; Wardle and Ng, 1999; Balbus and Terquem, 2001; Sano and Stone, 2002). In this section, we will discuss recent extensions of dynamo theory to include these effects.

\subsection{Ambipolar diffusion}

Ambipolar diffusion is important in the evolution of magnetic fields in protostars, proto-planetary circumstellar disks, as well as in the case of the galactic gas (Zweibel, 1988, 2002; Sano and Stone, 2002; Brandenburg and Subramanian, 2004). It is also relevant in the evolution of magnetic clouds (Zeldovichet al., 1983). In the interstellar medium, ambipolar diffusion is believed to be the principal mechanism responsible for breaking the frozen-in condition for the magnetic field.

The ambipolar drift occurs because the magnetic field lines are attached to the plasma ions but not to the neutrals. The Lorentz force acting over the magnetized ions generates a drift between the ions and the neutrals. If collisions are frequent, the Lorentz force acting on the ions is balanced by collisions with the neutrals. Under these assumptions, in a partially ionized medium with the bulk velocity $\boldsymbol{U}$ dominated by the neutrals, the induction equation (1) takes the form

$$
\frac{\partial \boldsymbol{B}}{\partial t}=\nabla \mathbf{x}[(\boldsymbol{U}+\lambda \boldsymbol{J} \times \boldsymbol{B}) \times \boldsymbol{B}]+\eta \nabla^{2} \boldsymbol{B},
$$

where $\lambda=\left(\rho_{i} v_{i n}\right)^{-1}$. Here, $\rho_{i}$ is the ion mass density, and $v_{i n}$ is the collision frequency between ions and neutrals. Note that in this approximation $\boldsymbol{U}_{i}=\boldsymbol{U}+\lambda \boldsymbol{J} \times \boldsymbol{B}$ is the velocity of the ions, and Eq. (16) expresses that the magnetic field lines in the ideal case (i.e. $\eta=0$ ) are frozen to the ion velocity field.

Simulations with this modified version of the induction equation have been made within the context of reconnection in the interstellar medium (Zweibel and Brandenburg, 1997), and the generation of sharp fronts together with a change in the reconnection rate of magnetic fields were observed. The effect of ambipolar diffusion in the dynamo was studied using a simplified model by Zweibel (1988), and an $\alpha$-effect was recovered from Eq. (16). It was shown that helical turbulence can amplify a magnetic field under these conditions, and ambipolar diffusion can alleviate the need of large turbulent diffusivity in some numerical models of galactic dynamos. More recently Brandenburg and Subramanian (2000) confirmed the existence of an $\alpha$-effect contributed by ambipolar diffusion, using both direct simulations in a periodic box and a closure model. 


\subsection{The Hall effect}

The Hall effect is relevant in dense molecular clouds (Wardle and $\mathrm{Ng}, 1999$ ), in accretion disks (including proto-planetary disks) (Wardle, 1999; Balbus and Terquem, 2001; Sano and Stone, 2002), in white dwarfs and neutron stars (Yakovlev and Urpin, 1980; Muslimov, 1994; Geppert and Rheinhardt, 2002; Geppertet et al., 2003), and in the early universe (Tajima et al., 1992). Recently, the impact of the Hall current on the dynamo effect was also measured in the laboratory (Ding et al., 2004).

With the inclusion of the Hall effect, the induction equation (1) reads

$$
\frac{\partial \boldsymbol{B}}{\partial t}=\nabla \mathbf{x}[(\boldsymbol{U}-\varepsilon \boldsymbol{J}) \mathbf{x} \boldsymbol{B}]+\eta \nabla^{2} \boldsymbol{B}
$$

The Hall term $\varepsilon \boldsymbol{J} \times \boldsymbol{B}$ is the manifestation of the difference in velocity between ions and electrons. Indeed, in a twospecies quasi-neutral electron-ion plasma $\boldsymbol{U}_{e}=\boldsymbol{U}-\varepsilon \boldsymbol{J}$ is the electron velocity. From Eq. (17), when $\eta=0$ the magnetic field is frozen to the velocity field, instead of the bulk velocity $\boldsymbol{U}$ as is the case in one-fluid MHD.

Assuming the characteristic velocity equal to the Alfvén velocity, the intensity of the Hall effect is given by $\varepsilon=c /\left(\omega_{p i} L_{0}\right)$. Here $c$ is the speed of light, and $\omega_{p i}$ is the ion plasma frequency. Note that $c / \omega_{p i}$ has dimensions of length (the ion skin depth), and corresponds to the lenghtscale where the Hall effect becomes non-negligible. General expressions for $\varepsilon$, as well as characteristic values for astrophysical objects can be found in Mininni et al. (2003).

Since in Eq. (17) the magnetic field is stretched by the electron velocity field $\boldsymbol{U}_{e}$ rather than the bulk velocity field $\boldsymbol{U}$, and these velocities can be quite different, the Hall term is expected to impact on dynamo mechanisms. The influence of the Hall effect on the dynamo was studied in simplified models by Heintzmann (1983), and by Galanti et al. (1994).

Using mean field theory, Mininni et al. (2002) showed that a helical flow can amplify a magnetic field in the Hall-MHD framework, and obtained a generalized expression for the $\alpha$ effect. The Hall term was found to enhance or suppress dynamo action depending on the value of $\varepsilon$. DNS of Hall-MHD helical dynamo action confirmed this result (Mininni et al., 2003, 2004).

The saturation amplitude of the Hall-MHD dynamo has a nonlinear dependence with the amplitude of the Hall effect $\varepsilon$ (Fig. 13). When the Hall lengthscale is close to or smaller than the Kolmogorov's magnetic dissipation scale $k_{\eta}=\left(\left\langle J^{2}\right\rangle / \eta^{2}\right)^{1 / 4}$, the MHD behavior is recovered $(\varepsilon \ll 1)$ and no differences in the evolution and saturation of the dynamo can be identified (the "MHD dynamo"). On the other hand, when the Hall effect dominates in all the relevant scales including the energy containing scales of the turbulent flow ( $\varepsilon \approx 1$, the so called "massive Hall-MHD dynamo"), the helical dynamo is less efficient than its MHD counterpart. The dynamo saturates at a lower level of total magnetic energy, and the kinetic energy dominates the dynamics at almost all scales.

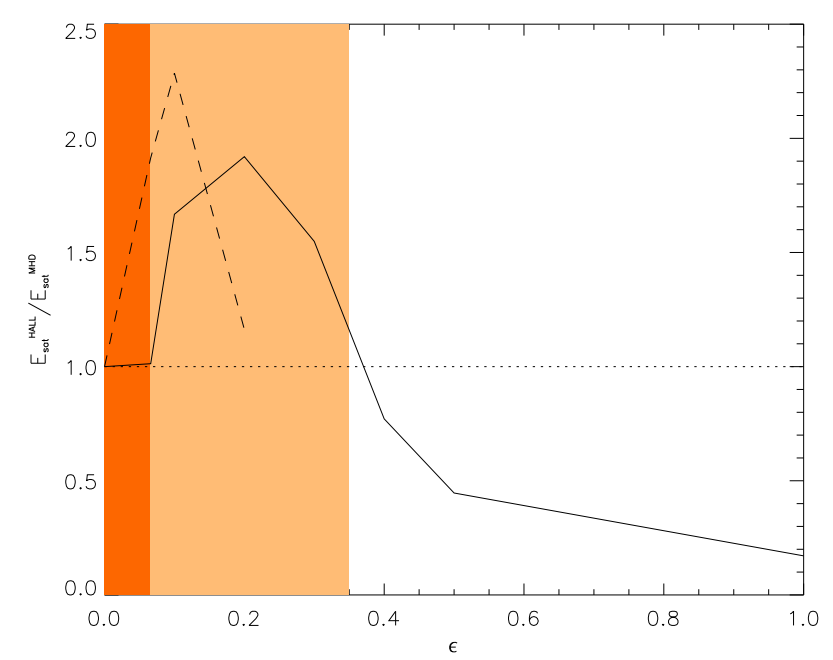

Fig. 13. Total magnetic energy in the saturated state of the HallMHD dynamo, as a function of the amplitude $\varepsilon$ of the Hall-effect. The energy is normalized by the saturated state of an MHD simulation with the same Reynolds numbers ( $R=100$ : solid line, $R=300$ : dashed line). The horizontal dotted line is showed as the MHD reference (corresponding to $\varepsilon=0$ ). The red area indicates the region where the dynamo asymptotically goes to the MHD solutions, and the orange area corresponds to the region where the dynamo effect is enhanced by the Hall current.

However, there is an intermediate regime in which the dynamo effect is enhanced by the Hall term (the "Halldominated microscale dynamo"). In this regime, the Hall effect dominates the dynamics at intermediate scales up to the diffusion. The enhancement was observed to increase as the Reynolds numbers and the scale separation are increased (Mininni et al., 2004), and the peak of maximum magnetic energy moves to smaller values of $\varepsilon$ as $R$ is increase. Also, in this regime the Hall effect was observed to alleviate the slow saturation of the MHD helical dynamo, and large scale magnetic fields grow faster than its MHD counterpart.

\section{Discussion}

In this work we presented direct simulations of helical MHD flows, using periodic boundary conditions and assuming incompressibility. Using these simulations, we studied the amplification and saturation of helical MHD dynamos and reviewed recent results.

For a flow with net helicity, the kinetic helicity injected at $k_{\text {force }}$ cascades down to smaller scales with its spectrum being proportional to $k_{\text {force }} E(k)$ (Chen et al., 2003; Chenet et al., 2003; Gómez and Mininni, 2004). We found that although all scales in Fourier space are dominated by the sign of helicity injected by the external force, in real space helicity displays strong spatial fluctuations. Also, regions with large relative kinetic helicity are strongly concentrated in space, forming elongated structures. 
In simulations of helical dynamos, we reviewed the detailed evolution of magnetic energy and magnetic helicity during the amplification and saturation stages. As we increased the Reynolds number, we found that the growth rate and the saturation level of the magnetic energy and the magnetic helicity seems to reach an asymptotic value. This behavior was also found in the evolution of the magnetic energy by Archontis et al. (2003).

Finally, we reviewed recent extensions made to the standard MHD dynamo theory, to include kinetic effects relevant in several astrophysical scenarios, such as protostars, protoplanetary disks, the interstellar medium, white dwarfs, and neutron stars. The most important extensions to the theory in these objects are ambipolar diffusion and the Hall effect.

The idealized conditions used in these dynamo simulations are far from astrophysical or geophysical applications. Also, the values of the Reynolds numbers considered here are far away from realistic values for astrophysical or geophysical flows. This is even more relevant when extensions to one-fluid MHD are considered. The study of new effects (as ambipolar drift or the Hall effect) introduces new lenghtscales, making the separation between physically relevant scales (e.g. the energy injection band, the Hall scale, and the dissipation scale) even harder. However, despite the fact that these studies are still performed under idealized conditions, the results are encouraging. The inclusion of new effects in MHD dynamo mechanisms, can help the development of the theory under conditions more appropriate for astrophysical plasmas.

Acknowledgements. Use of computer time at the Bocha Beowulf cluster at the Departamento de Física (FCEN, UBA) is acknowledged. Research of DOG has been partially founded by grant X209/01 from the University of Buenos Aires and by grant PICT 03-9483 from ANPCyT. DOG is a member of the Carrera del Investigador Científico of CONICET.

Edited by: P.-L. Sulem

Reviewed by: two referees

\section{References}

Archontis, V., Dorch, S. B. F. and Nordlund, Å.: Dynamo action in turbulent flows, Astron. \& Astrophys., 410, 759-766, 2003.

Armstrong, J. W., Rickett, B. J. and Spangler, S. R.: Electron density power spectrum in the local interstellar medium, Astrophys. J., 443, 209-221, 1995.

Balbus, S. A. and Terquem, C.: Linear Analysis of the Hall effect in protostellar disks, Astrophys. J., 552, 235-247, 2001.

Balsara, D. and Pouquet, A.: The formation of large scale structures in supersonic magnetohydrodynamic flows, Phys. Plasmas, 6, 89-99, 1999.

Blandford, R. D. and Payne, D.G.: Hydromagnetic flows from accretion discs and the production of radio jets, Monthly Not. R. Astr. Soc., 199, 883-903, 1982.

Borue, V. and Orzag, A.: Spectra in helical three-dimensional homogeneous isotropic turbulence, Phys. Rev. E, 55, 7005-7009, 1997.
Brandenburg, A.: The inverse cascade and nonlinear alpha-effect in simulations of isotropic helical hydromagnetic turbulence, Astrophys. J., 550, 824-840, 2001.

Brandenburg, A. and Dobler, W.: Large scale dynamos with helicity loss through boundaries, Astron. \& Astrophys., 369, 329-338, 2001.

Brandenburg, A. and Dobler, W.: Solar and stellar dynamos - latest developments, Astron. Nachr., 323, 411-416, 2002.

Brandenburg, A. and Subramanian, K.: Astrophysical magnetic fields and nonlinear dynamo theory, arXiv:astro-ph/0405052, 2004.

Brandenburg, A. and Subramanian, K.: Large scale dynamos with ambipolar diffusion nonlinearity, Astron. \& Astrophys., 361, L33-L36, 2000.

Braginsky, S. I.: Magnetogidrodinamika zemnovo yadra, Geomagnetizm i Aeronomiya, 4, 989-916, 1964.

Brandenburg, A., Saar, S. H. and Turpin, C. R.: Time evolution of the magnetic activity cycle period, Astrophys. J. Lett., 498, L51L54, 1998.

Brissaud A., Frisch, U., Léorat, J., Lesieur, M., and Mazure, A.: Helicity cascades in fully developed isotropic turbulence, Phys. Fluids, 16, 1366-1367, 1973.

Buffett, B. A.: Earth's core and the geodynamo, Science, 288, 2007-2012, 2000.

Bullard, E.: The magnetic field within the Earth, Proc. Roy. Soc. London, A197, 433-453, 1949.

Canuto, C., Hussaini, M. Y., Quarteroni, A., and Zang, T. A.: Spectral Methods in Fluid Dynamics, Springer-Verlag, New York, 1988.

Casse, F. and Keppens, R.: Radiatively inefficient magnetohydrodynamic accretion-ejection structures, Astrophys. J., 601, 90-103, 2004.

Cattaneo, F. and Hughes, D. W.: Nonlinear saturation of the turbulent $\alpha$ effect, Phys. Rev. E, 54, R4532-R453, 1996.

Cattaneo, F.: On the origin of magnetic fields in the quiet photosphere, Astrophys. J. Lett., 515, 39-42, 1999.

Chen, Q., Chen, S., and Eyink, G. L.: The joint cascade of energy and helicity in three-dimensional turbulence, Phys. Fluids, 15, 361-374, 2003a.

Chen, Q., Chen, S., Eyink, G. L., and Holm, D.D.: Intermittency in the joint cascade of energy and helicity, Phys. Rev. Lett., 90, 214503, 2003b.

Childress, S.: New solutions of the kinematic dynamo problem, J. Math. Phys., 11, 3063-3076, 1970.

Dikpati, M. and Charbonneau, P.: A Babcock-Leighton flux transport dynamo with solar-like differential rotation, Astrophys. J., 518, 508-520, 1999.

Ding, W. X., Brower, D. L., Craig, D., Deng, B. H., Fiksel, G., Mirnov, V., Prager, S. C., Sarff, J. S., Svidzinski, V.: Measurement of the Hall dynamo effect during magnetic reconnection in a high-temperature plasma, Phys. Rev. Lett., 93, 045002, 2004.

Espagnet, O., Muller, R., Roudier, T., and Mein, N.: Turbulent power spectra of solar granulation, Astron. \& Astrophys., 271, 589-600, 1993.

Galanti, B., Sulem, P.-L., and Gilbert, A. D.: Inverse cascades and time-dependent dynamos in MHD flows, Physica D, 47, 416426, 1991.

Galanti, B., Sulem, P.-L., and Pouquet, A.: Linear and non-linear dynamos associated with ABC flows, J. Geophys. Astrophys. Fluid Dyn., 66, 183-208, 1992.

Galanti, B., Kleeorin, N., and Rogachevskii, I.: Nonlinear dynamo in ABC flow: the Hall effect, Phys. Plasmas, 1, 3843-3849, 
1994.

Geppert, U. and Rheinhardt, M.: Non-linear magnetic field decay in neutron stars, Theory and observations, Astron. \& Astrophys., 392, 1015-1024, 2002.

Geppert, U. Rheinhardt, M. and Gil, J.: Spot-like structures of neutron star surface magnetic fields, Astron. \& Astrophys., 412, L33-L36, 2003.

Gilbert, A. D.: Magnetic helicity in fast dynamos, Geophys. Astrophys. Fluid Dynam., 96, 135-151, 2002.

Glatzmaier, G. A., Coe, R. S., Hongre, L., and Roberts, P. H.: The role of the Earth's mantle controlling the frequency of geomagnetic reversals, Nature, 401, 885-890, 1999.

Gómez, D. O., and Mininni, P. D.: Understanding turbulence through numerical simulations, Physica A, 342, 69-75, 2004.

Gómez, D. O., Mininni, P. D. and Dmitruk, P.: Parallel simulations in turbulent MHD, Adv. Space Res., in press, 2004.

Haugen, N. E. L., Brandenburg, A. and Dobler, W.: Is nonhelical hydromagnetic turbulence peaked at small scales?, Astrophys. J. Lett., 597, 141-144, 2003.

Haugen, N. E. L., Brandenburg, A. and Dobler, W.: Simulations of nonhelical hydromagnetic turbulence, Phys. Rev. E, 70, 016308, 2004.

Heintzmann, H.: The Hall effect in a unipolar inductor - A possible mechanism for a dynamo and an antidynamo, J. Exp. Theor. Phys., 57, 251-259, 1983.

Hughes, D. W., Cattaneo, F., and Kim, E. J.: Kinetic helicity, magnetic helicity and fast dynamo action, Phys. Lett. A, $223167-$ 172, 1996.

Kazantsev, A. P.: Enhancement of a magnetic field by a conducting fluid, Sov. Phys. JETP, 26, 1031-1034, 1968.

Kraichnan, R. H.: Helical turbulence and absolute equilibrium, J. Fluid Mech., 59, 745-752, 1973.

Krause, F. and Rädler, K.-H.: Mean-Field Magnetohydrodynamics and Dynamo Theory, Pergamon Press, New York, 1980.

Krishan, V., Paniveni, U., Singh, J., and Srikanth, R.: Relationship between horizontal flow velocity and cell size for supergranulation using SOHO Dopplergrams, M. Not. R. Ast., 334, 230-232, 2002.

Larmor, J.: How could a rotating body such as the sun become a magnet?, Brit. Assn. Adv. Sci. Rep., 1919, 159-160, 1919.

Leighton, R. B.: A magneto-kinematic model of the solar cycle, Astrophys. J., 156, 1-26, 1969.

Matsumoto, R., Uchida, Y., Hirose, S., et al.: Radio Jets and the Formation of Active Galaxies: Accretion Avalanches on the Torus by the Effect of a Large-Scale Magnetic Field, Astrophys. J., 461, 115-126, 1996.

Meneguzzi, M., Frisch, U., and Pouquet, A.: Helical and nonhelical turbulent dynamos, Phys. Rev. Lett., 47, 1060-1064, 1981.

Meneguzzi, M. and Pouquet, A.: Turbulent dynamos driven by convection, J. Fluid. Mech., 205, 297-318, 1989.

Mininni, P. D., Gómez, D. O., and Mahajan, S. M.: Dynamo action in Hall magnetohydrodynamics, Astrophys. J. Lett., 567, 81-83, 2002.

Mininni, P. D., Gómez, D. O., and Mahajan, S. M.: Dynamo action in magnetohydrodynamics and Hall-magnetohydrodynamics, Astrophys. J., 587, 472-481, 2003.

Mininni, P. D., Gómez, D. O., and Mahajan, S. M.: Direct Simulations of Helical Hall-MHD Turbulence and Dynamo Action, Astrophys. J., in press, 2004.
Minter, A. H. and Spangler, S. R.: Observation of turbulent fluctuations in the interstellar plasma density and magnetic field on spatial scales of 0.01 to 100 parsecs, Astrophys. J., 458, 194-214, 1996.

Moffatt, H. K.: Magnetic field generation in electrically conducting fluids, Cambridge University Press, Cambridge, 1978.

Muslimov, A. G.: Magnetic field evolution in neutron stars - coupling between poloidal and toroidal components in the crust, $\mathrm{M}$. Not. R. Ast., 267, 523-528, 1994.

Nore, C., Brachet, M. E., Politano, H., and Pouquet, A.: Dynamo action in the Taylor-Green vortex near threshold, Phys. Plasmas Lett., 4, 1-3, 1997.

Parker, E. N.: Hydromagnetic dynamo models, Astrophys. J., 122, 293-314, 1955.

Podvigina, O., and Pouquet, A.: On the non-linear stability of the 1:1:1 ABC flow, Physica D, 75, 471-508, 1994.

Pouquet, A., Frisch, U., and Léorat, J.: Strong MHD helical turbulence and the nonlinear dynamo effect, J. Fluid Mech., 77, 321354, 1976.

Priest, E. and Forbes, T.: Magnetic reconnection: MHD theory and applications. Cambridge University Press, Cambridge, 2000.

Sano, T. and Stone, J. M.: The effect of the Hall term on the nonlinear evolution of the magnetorotational instability, I. Local axisymmetric simulations, Astrophys. J., 570, 314-328, 2002.

Schekochihin, A., Cowley, S., Maron, J., and Malyshkin, L.: Structure of small-scale magnetic fields in the kinematic dynamo theory, Phys. Rev. E, 65, 016305, 2001.

Schekochihin, A .A., Cowley, S. C., Taylor, S. F., Hammett, G. W., Maron, J. L., and McWilliams J. C.: Saturated state of the nonlinear small-scale dynamo, Phys. Rev. Lett., 92, 084504, 2004.

Seehafer, N.: Nature of the $\alpha$ effect in magnetohydrodynamics, Phys. Rev. E, 53, 1283-1286, 1996.

Steenbeck, M., Krause, F., and Rädler, K.-H.: Berechnung der mittleren Lorentz-Feldstärke $\overline{\boldsymbol{v} \times \boldsymbol{B}}$ für ein elektrisch leitendendes Medium in turbulenter, durch Coriolis-Kräfte beeinflußter Bewegung, Z. Naturforsch., 21a, 369-376, 1966.

Tajima, T., Cable, S., Shibata, K., and Kulsrud, R. M.: On the origin of cosmological magnetic fields, Astrophys. J., 390, 309-321, 1992.

Tobias, S.: Relating stellar cycle periods to dynamo calculations, Monthly Not. R. Astr. Soc., 296, 653-661, 1998.

Urpin, V.: Mean electromotive force in turbulent shear flow, Phys. Rev. E, 64, 026301, 2002.

Wardle, M.: The Balbus-Hawley instability in weakly ionized discs, M. Not. R. Ast., 307, 849-856, 1999.

Wardle, M. and Ng, C.: The conductivity of dense molecular gas, M. Not. R. Ast., 303, 239-246, 1999.

Yakovlev, D. G. and Urpin, V. A.: On thermal and electrical conductivities in neutron stars and white-dwarfs, Astr. Zh., 57, 526-536, 1980.

Zeldovich, Ya .B., Ruzmaikin, A. A., and Sokoloff, D. D.: Magnetic fields in astrophysics. Gordon and Breach, New York, 1983.

Zweibel, E. G.: Ambipolar diffusion drifts and dynamos in turbulent gases, Astrophys. J., 329, 384-391, 1988.

Zweibel, E. G. and Brandenburg, A.: Current sheet formation in the interstellar medium, Astrophys. J., 478, 563-568, 1997.

Zweibel, E. G.: Ambipolar drift in a turbulent medium, Astrophys. J., 567, 962-970, 2002. 